\title{
Quiet-Filament Eruptions and Coronal Jets as Causes of Depressions in Microwave Radio Emission
}

\author{
I. V. Kuzmenko ${ }^{a}$ * \\ ${ }^{a}$ Institute of Applied Astronomy, Russian Academy of Sciences, St. Petersburg, Russia \\ *e-mail: kuzmenko_irina@mail.ru
}

Received June 17, 2021; revised August 27, 2021; accepted August 30, 2021

\begin{abstract}
Several solar events with different types of negative microwave bursts have been studied using data from different spectral ranges. The total radio flux data obtained at the Ussuriysk Observatory, the Nobeyama Observatory, the US Air Force Radio Solar Telescope Network (RSTN), and the spectropolarimeter of the Institute of Solar-Terrestrial Physics, Siberian Branch of the Russian Academy of Sciences (ISTP SB RAS) were used. The images were analyzed using data from the SDO/AIA space observatory in the $304 \AA$ channel and the Nobeyama radio heliograph at a frequency of $17 \mathrm{GHz}$. It was shown that the "isolated" depressions of radio emission were caused by the absorption of radiation from radio sources and/or vast regions of the quiet Sun by low-temperature material of a large eruptive filament in the absence of flares. This confirmed the conclusions of the previous studies. It was revealed that the cause of negative bursts of the "pre-burst depression" type was the screening of a near-limb radio source by the material of coronal jets. In the case of a weak flare accompanying the jet, the negative burst could also be of the "isolated" type. A case of a previously unreported occurrence of a deeper depression of radio emission at high frequencies as compared to low frequencies was considered. It was shown that negative bursts are not as rare phenomena as previously thought.
\end{abstract}

Keywords: depression of radio emission, microwave negative burst, radio source, eruptive prominence, coronal jet

DOI: $10.1134 / \mathrm{S} 1063772921120040$

\section{INTRODUCTION}

The analysis of sporadic solar activity using microwave radio emission makes it possible to study eruptive events. Some of these events feature so-called negative radio bursts, which are a temporary decrease in the total radio flux below a quasi-stationary level outside the bursts. The classification of negative bursts was made in $[1,2]$ based on the records of radio emission at a frequency of $2.8 \mathrm{GHz}$, where they were first detected. The following types are distinguished: preburst depression (original name-negative precursor); depression overlapping a gradual burst; isolated burst (absorption); and the most common and well-studied-post-burst depression (post burst decrease). Negative bursts are considered quite rare: only 12 such events were recorded in Ottawa for the period of 19511972 and 67 events for the period of 1991-2010 according to Solar Geophysical Data [3].

The study of some negative burst events based on data from different spectral ranges showed that the depressions of radio emission were mainly caused by the absorption of radiation from local radio sources and vast regions of the quiet Sun by low-temperature plasma of an eruptive filament [1-6]. Negative bursts also occurred as a result of screening of bright near- limb radio sources by the material of jet-like eruptions $[7,8]$. Recall that jets are small-scale solar plasma ejections observed in the extreme ultraviolet (EUV) and X-ray ranges. Another possible cause for negative bursts is a decrease in radiation from a radio source during the development of an active region $[9,10]$.

The dependence of radio absorption on the parameters of the absorbing matter and the frequency of the recorded radio emission allows us to estimate the parameters of the absorber based on radio data at several frequencies. The method for estimating the parameters of the eruption from the magnitude of radio absorption is described in [11]; it consists in modeling the spectral variation of the total solar radio flux in the presence of an absorbing medium on the way to the observer, and in searching for such parameters of the eruption plasma that lead to the best correspondence to the radio flux values measured at different frequencies. The model calculations for a number of events showed that the radio-absorbing material had a temperature $\sim 10^{4} \mathrm{~K}$ and occupied an area less than $10 \%$ of the solar disk area [3]. The estimated masses of the absorber were comparable to the masses of large prominences. 


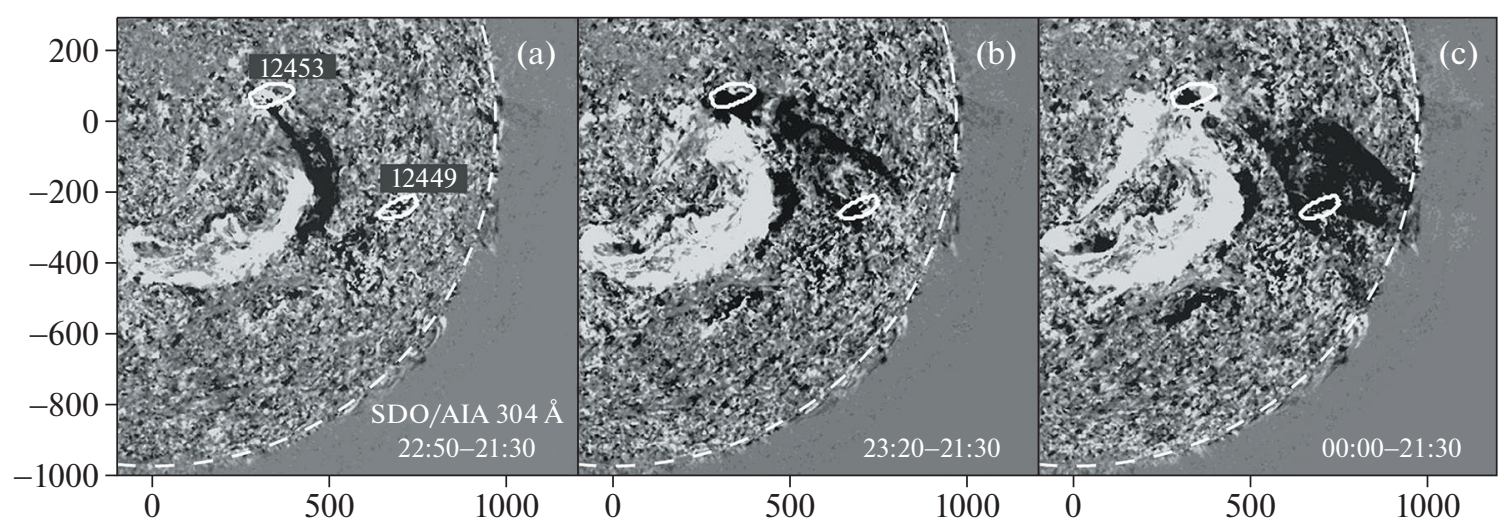

Fig. 1. Filament eruption on November 15, 2015 in the difference SDO/AIA images in the $304 \AA$ channel. The outlines show the positions of radio sources above the active regions determined from the images of the Nobeyama radioheliograph at a frequency of $17 \mathrm{GHz}$. Hereinafter, the coordinate axes show angular seconds from the center of the solar disk.

This paper is dedicated to clarifying the causes of negative bursts in several events selected from the records of the total radio flux at a frequency of $2.8 \mathrm{GHz}$ obtained at the Ussuriysk Astrophysical Observatory [12] (currently, Institute of Applied Astronomy, Russian Academy of Sciences). In the first event (November 15-16, 2015), two "isolated" negative bursts were studied; in other events (September 7-9, 2017), depressions of different types were considered, but negative bursts of the "pre-burst absorption" type were examined in more detail. Section 2 presents the analysis of observations of events in different spectral ranges, as well as the estimate of the parameters of the absorbing matter for one of the events. Section 3 is dedicated to the discussion of the results; Section 4 presents the main findings of the study.

The main sources of information were various Internet data centers. The records of total radio fluxes from Nobeyama radio polarimeters ${ }^{1}$ and the stations of the US Air Force Radio Solar Telescope Network $(\text { RSTN })^{2}$ were used. The observations in the EUV range were analyzed using the images obtained by the AIA instrument of the SDO solar space observatory, ${ }^{3}$ and in the radio range using the images of the Nobeyama radio heliograph. ${ }^{4}$ Coronal ejection data were taken from the SOHO LASCO CME Catalog. ${ }^{5}$

\section{ANALYSIS OF THE OBSERVATIONS}

\subsection{Event of November 15-16, 2015}

Two "isolated" negative bursts were detected on November 15-16, 2015 in the recording of the total

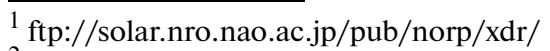

2 ftp://ftp.ngdc.noaa.gov/STP/SOLAR_DATA/SOLAR_RADIO/RSTN_1sec/

${ }_{3}^{3} \mathrm{http}: / /$ jsoc.stanford.edu/data/aia/synoptic/

${ }_{5}^{4} \mathrm{http}: / /$ solar.nro.nao.ac.jp/norh/images/10min/

5 http://cdaw.gsfc.nasa.gov/CME_list/
}

radio flux at a frequency of $2.8 \mathrm{GHz}$ according to the Ussuriysk Observatory data. Although the beginning of observations on November 15 at 23:15 coincided with the already ongoing first depression of radio emission, we can assume it to be an "isolated" negative burst, since there were no flares until 23:00 according to the GOES SXR $1-8 \AA$ Aata.

The event was associated with two eruptions of quiet filaments in the southwestern quadrant of the solar disk; one of the eruptions was sympathetic. The first large eruptive filament was located between the active regions NOAA 12452 (S08, W13) and 12449 (S11, W49) of the magnetic class $\beta$ with small sunspots. The rise and expansion of the filament was observed from 21:30 both in the $\mathrm{H} \alpha$ line (e.g., in MLSO images) and in extrime ultraviolet channels in SDO/AIA images. A possible cause of eruption is the emergence and cancellation of magnetic flux of opposite polarity, which occurred in several places along the neutral line of the magnetic field [13, 14]. The eruption was accompanied by flare-like ribbons visible in the EUV and a slowing coronal mass ejection (CME).

SDO/AIA images in the $304 \AA$ channel (maximum temperature sensitivity $80000 \mathrm{~K}$ ) showed the heating of the filament plasma at the beginning of eruption at the sites of breakaway of its legs, and screening by its matter of vast quiet-Sun regions and active regions located to the north and west of its initial position (Fig. 1). The analysis of the Nobeyama radioheliograph images at $17 \mathrm{GHz}$ confirmed that, during its rise and expansion, the filament obscured the radiation of two thermal radio sources located above the active regions NOAA 12453 and 12449. Their position is shown with the white outlines in Fig. 1.

Figure 2 presents the flux values measured from radio images at $17 \mathrm{GHz}$ of these sources in units of sfu $\left(1 \mathrm{sfu}=10^{-22} \mathrm{~W} /\left(\mathrm{m}^{2} \mathrm{~Hz}\right)\right)$. In the calculations of these values, the brightness temperature of the quiet Sun was subtracted within the area of the radio source. 


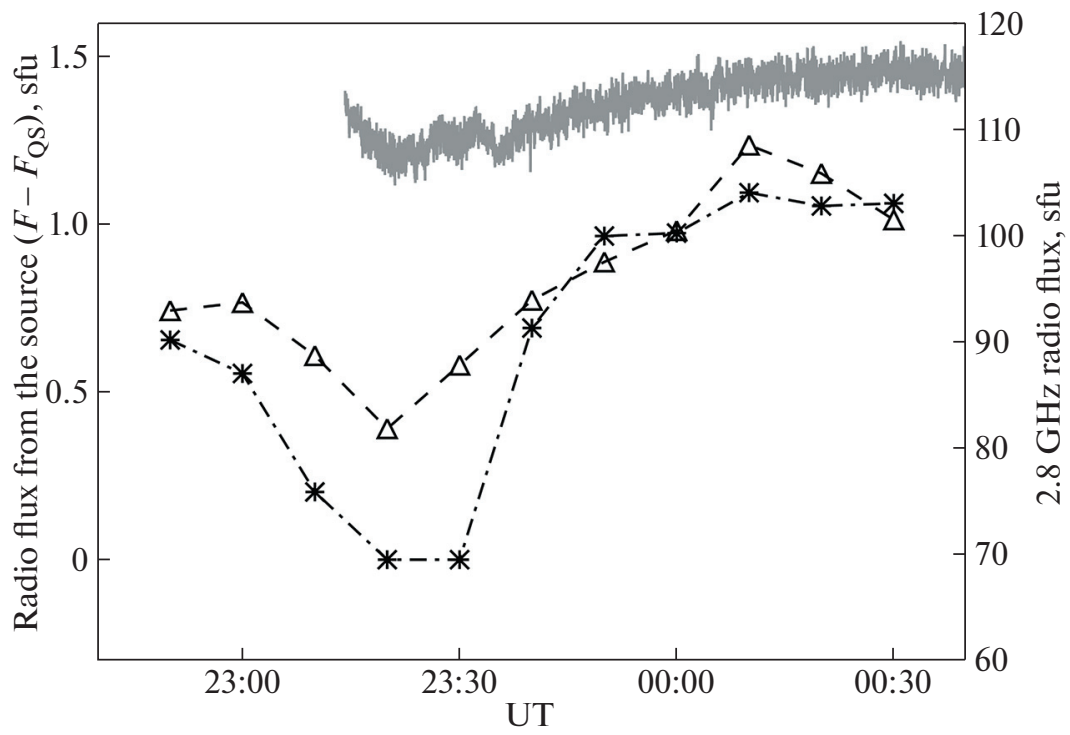

Fig. 2. The flux from radio sources over the active regions NOAA 12453 (asterisks) and 12449 (triangles) measured on November 15-16, 2015 from the images of the Nobeyama radioheliograph at $17 \mathrm{GHz}$. The solid gray line shows the record of the total radio flux at a frequency of $2.8 \mathrm{GHz}$.

Figure 2 also shows the record of the $2.8 \mathrm{GHz}$ total radio flux from the data of the Ussuriysk Observatory. It can be seen that the isolated negative burst in the recording of the total radio flux coincides in time with a decrease in the flux from the sources. A similar depression of radio emission usually occurs at other microwave frequencies as well; however, these data (for example, from the RSTN network) could not be found on the Internet. Observations at the Nobeyama radio polarimeters began only at 00:00 on November 16.

Similar events with radio emission depressions observed at a number of microwave frequencies were studied by us earlier $[3,5,6]$. By analogy with the previous results, we conclude that the cause of the "isolated" negative burst in this case was the absorption of radio emission from both the quiet-Sun regions and radio sources by the material of the eruptive filament. This was the eruption of a large quiet filament located between two active regions.

The second "isolated" negative burst was observed at microwave frequencies of $2.8-9.4 \mathrm{GHz}$ according to data from different observatories. We used the records of the Nobeyama radio polarimeters, the Ussuriysk radiometer, and the ISTP SB RAS spectropolarimeter in the frequency range of 4-8 GHz [15]. Figure $3 \mathrm{a}$ shows the radio emission profiles smoothed over 3-20 s with the subtracted pre-burst level $F_{\text {before }}$ and normalized to the level of the quiet Sun $F_{\mathrm{QS}}$ (see below about the necessity of normalization). The $F_{\mathrm{QS}}$ values at these frequencies were determined by approximating the measured values for the quiet Sun at a number of frequencies obtained in [16].
The vertical dash-dotted line shows the maximum absorption at 02:24.

During the second negative burst, there was a partial sympathetic eruption of another quiet filament, which was located southeast of the active regions NOAA 12449 and 12450. The eruption also led to the appearance of flare-like ribbons observed in the EUV and a fast CME. The analysis of the SDO/AIA images in EUV and images of the Nobeyama radioheliograph at a frequency of $17 \mathrm{GHz}$ showed that during its rise the filament material screened only the regions of the quiet Sun (Fig. 4).

In [11], a model was proposed for estimating the parameters of the absorbing matter from the measured values of radio absorption at different frequencies. The measurements were carried out using radio emission profiles normalized to the level of the quiet Sun, which are shown in Fig. 3a. In the calculations, the parameters of the absorber were selected so that the calculated values of radio absorption were close to the measured values at different frequencies. The case when only the radiation of the quiet-Sun regions was absorbed was the simplest. In Fig. 3b, the asterisks show the absorption values measured at 02:24; the solid line shows the values calculated using the model. The calculated curve was obtained with the following parameters of the absorbing matter: its kinetic temperature is $9000 \mathrm{~K}$, the optical depth at a frequency of $17 \mathrm{GHz}$ is 0.08 , the height of the lower boundary of the absorbing screen above the chromosphere is $20 \mathrm{Mm}$, and the area of the screen is $8 \%$ of the solar disk area. Additional use of the ISTP spectropolarimeter data in this case allowed us to reduce the uncertainty of the calculated curve in the frequency range of 4-8 GHz. Using the values of 


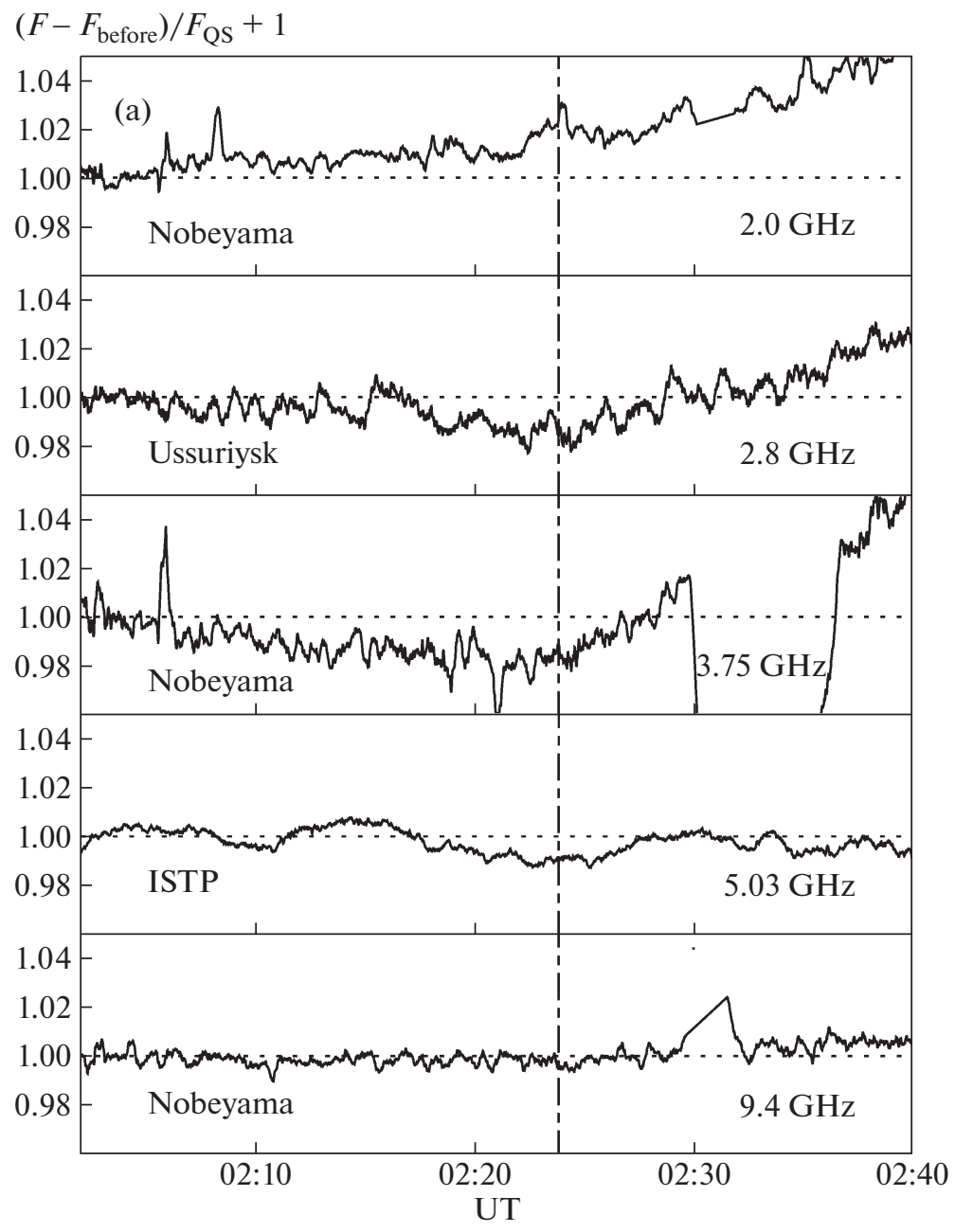

$\left(F-F_{\text {before }}\right) / F_{\mathrm{QS}}+1$

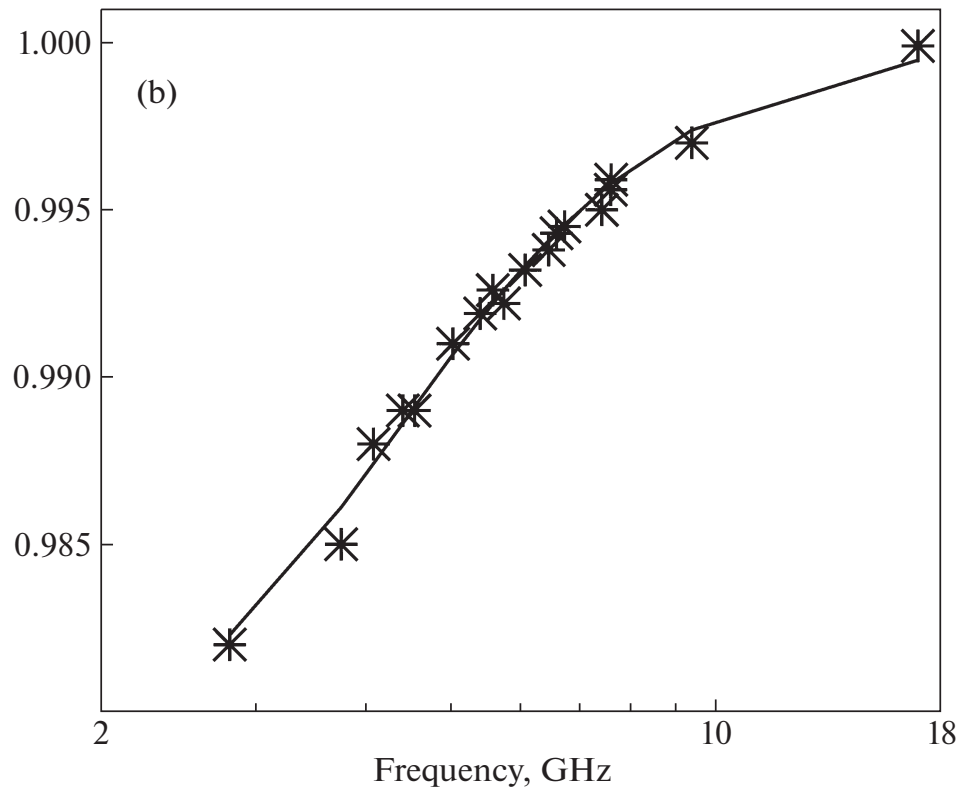

Fig. 3. (a) Normalized to the level of the quiet Sun $F_{\mathrm{QS}}$ profiles of radio emission at different frequencies with subtracted preburst level $F_{\text {before }}$. The vertical dash-dotted line marks the maximum absorption depth for which the measurements were made. (b) Spectrum of the depth of radio absorption at 02:24. Asterisks show the measured values, solid line shows the model approximation. 


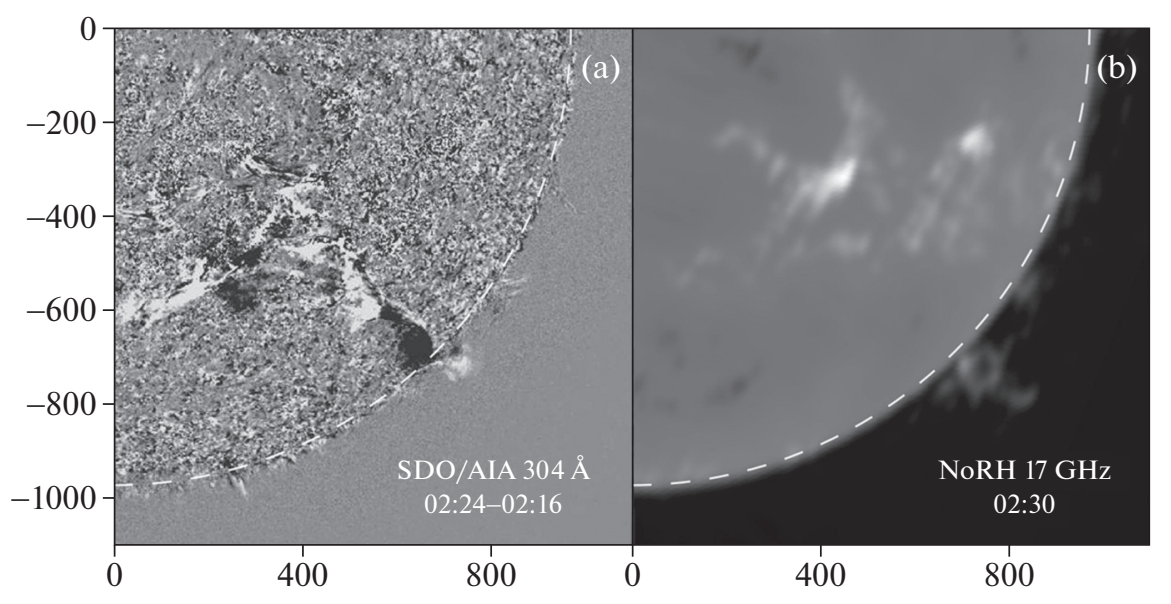

Fig. 4. Eruptive filament of November 16, 2015: (a) in the difference SDO/AIA image in the $304 \AA$ channel; (b) in the image of the Nobeyama radio heliograph at a frequency of $17 \mathrm{GHz}$ (above the limb).

the estimated parameters and the method described in [11], we also obtained an estimate of the mass of the absorber, $3.3 \times 10^{15} \mathrm{~g}$. The obtained parameters of the absorbing matter confirm that the second "isolated" negative burst in the event of November 15-16, 2015 was caused by the absorption of radio emission only from the quiet-Sun regions by the low-temperature material of the eruptive filament.

Thus, the analysis of observations in different spectral ranges showed that the two "isolated" negative bursts in the event of November 15-16, 2015 were caused by the absorption of radio emission in the cold matter of large filaments during their eruption. In the first case, both the quiet-Sun regions and the neighboring radio sources were screened; in the second case, radio emission was only absorbed from the quietSun regions.

\subsection{Events of September 7-9, 2017}

Two negative bursts of the "pre-burst absorption" type were revealed on September 8-9, 2017 from the records of $2.8 \mathrm{GHz}$ radio emission at the Ussuriysk Observatory. They were also observed at different frequencies at several observatories, what confirmed their solar origin. The third depression of radio emission was recorded outside the time interval of Ussuriysk observations; it was studied in [7].

Figure 5 shows smoothed radio emission records from the data of different observatories with the subtracted value of the pre-burst radiation level $F_{\text {before }}$. Due to the high instability, the record from the Learmonth station at a frequency of $8.8 \mathrm{GHz}$ was replaced by a record of another RSTN station, San Vito, where observations had just begun. The three time points marked in Fig. 5 with vertical dashed lines refer to depressions of radio emission. The panels with the radio flux records at 5.0 and $8.8 \mathrm{GHz}$, next to the radio bursts, show the classes of the corresponding flares according to the GOES SXR 1-8 A data. All the flares occurred in the active region NOAA 12673, which was located near the solar limb (coordinates S09, W82) on September 9, 2017. During its development from September 1 to 5, 2017, its magnetic configuration changed from $\alpha$ to $\beta \gamma \delta$ and after passing the central meridian of the Sun, it showed high activity in the form of multiple flares, including class X. It can be seen in Fig. 5 that the M-class flares had responses in the form of radio bursts at all frequencies, while weaker flares corresponded to bursts only at high frequencies. In this regard, the type of the third negative burst is uncertain; it can be attributed to both the "isolated" type and the "post-burst absorption" type.

As shown in [7], the cause for this deepest depression at all frequencies (after 07:00) was the screening of microwave sources over sunspots by the material of a small eruptive filament observed as a jet. The analysis of the original SDO/AIA images in the 304 Å channel showed that jets were also observed in or near the active region during the other two negative bursts. Figure 6 shows the difference images of a part of the solar disk in the $304 \AA$ channel at the time points marked in Fig. 5 by vertical dashed lines, as well as at 06:00. The position of the radio source determined from the images of Nobeyama radioheliograph at a frequency of $17 \mathrm{GHz}$ on September 8 at 23:00 at intensity levels of 0.5 and 0.9 from the maximum is shown by the outline in Fig. 6 considering the rotation of the Sun at the time indicated on the panels.

The jet of September 8 (Fig. 6a) was observed in the images in the $304 \AA$ A channel at 23:08. Its material partially screened the microwave source. After the second jet (Fig. 6b), according to the SOHO/LASCO catalog on September 9 at 06:12, a decelerating CME without a pronounced core was detected. At frequencies $\geq 5 \mathrm{GHz}$, a decrease in the level of the total radio flux 


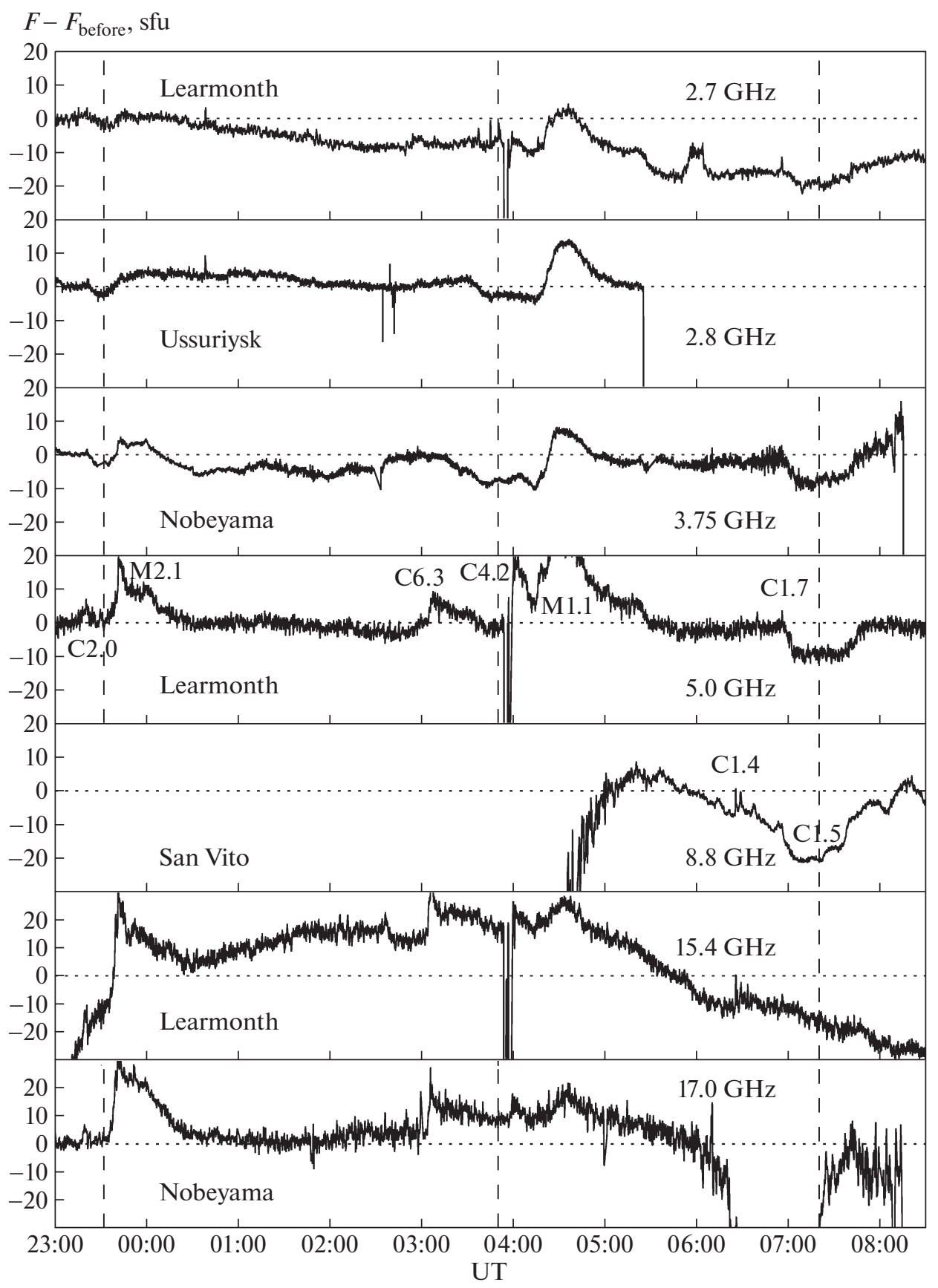

Fig. 5. Radio emission profiles on September 8-9, 2017 at different frequencies with the subtracted pre-burst level $F_{\text {before }}$. The vertical dashed lines mark three depressions of radio emission.

is noticeable (Fig. 5), but due to the flare radiation, there are no negative bursts at these frequencies.

It should be noted that after 05:40, the filament activation was observed in the SDO/AIA images at $304 \AA$ (Fig. 6c) and the original images of the Nobeyama radioheliograph. Probably, when its material rose to a small height, partial screening of the brightest part of the radio source occurred, which manifested itself in a decrease in the level of radio emission only at frequencies $>5 \mathrm{GHz}$ (Fig. 5). The direction of motion of the third jet and its dimensions were such (Fig. 6d) that its material screened most of the radio source. This led to a depression of radio emission at all frequencies from 2.7 to $17 \mathrm{GHz}$.

The parameters of the absorbing material for this event were not estimated using the model, since the model considers the screening of the source of thermal braking radiation. When the depression of radio emis- 


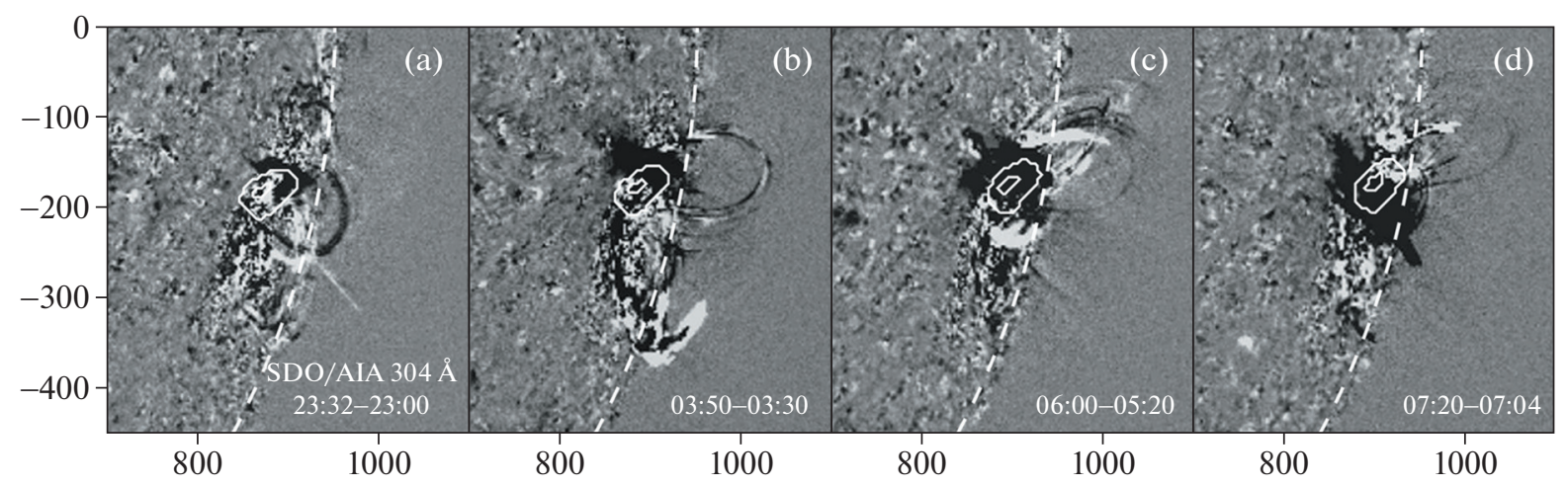

Fig. 6. Difference images of a part of the solar disk on September 8-9, 2017 according to the SDO/AIA data in the $304 \AA$ channel. The white outline shows the position of the radio source determined from the images of the Nobeyama radioheliograph at a frequency of $17 \mathrm{GHz}$ at the intensity level [0.5; 0.9] from the maximum; the dashed arc is the solar limb.

sion after 07:00 was studied in [7], it was noted that the material of the jet screened one or more polarized sources associated with sunspots, since the decrease in intensity had an analogue in the change in the polarization of radio emission at frequencies of $4-8 \mathrm{GHz}$. In addition, the authors of [17] showed that since September 5, 2017, the main component of the quasi-stationary emission of the active region of NOAA 12673 at a frequency of $17 \mathrm{GHz}$ was a compact source above the neutral line, whose brightness temperature may be higher than that of conventional gyroresonance sources.

It should be noted that since September 7, 2017, numerous jets have been detected in the $304 \AA$ A channel when active region approaching the western limb. After determining the time of their observations, it was found that most of them can be identified with depressions of radio emission at frequencies in the $1-17 \mathrm{GHz}$ range. Information about these negative bursts detected according to the data from different observatories, as well as about flares occurring in these time intervals, are given in Table 1.

Over two days (September 7 and 8), nine more negative bursts were revealed. Probably, there could be more, but some of the jets (for example, on September 8 at 05:54 and 08:57) were observed during powerful flares, against the background of which depressions of radio emission are usually not noticeable. In addition, 5 jets observed on September 7 and 8 from 17:00 to 22:00 occurred in the absence of radio observations (data from Sagamore Hill station, where observations are carried out during this time period, are not available on the RSTN website). Since the lifetime of the jets was tens of minutes, if they were observed directly one after another, there could be only one depression of radio emission (for example, nos. 1 and 9 in Table 1).

Thus, the analysis of images in different spectral ranges showed that the matter of jets that periodically appeared on September 7-9, 2017 in or near an active region with a complex magnetic configuration approaching the solar limb partially screened the radio sources (one or more) located in it, which led to numerous depressions of radio emission at a number of microwave frequencies.

\section{DISCUSSION}

Our study of events with different types of negative bursts using data from different spectral ranges confirmed our earlier conclusions regarding the causes of depressions in radio emission. Nevertheless, each event was unique and had its own specific features. In the event of November 15, 2015, the eruption of a large filament at first glance looked anomalous [18], when the material of the eruptive filament during eruption does not retain its shape and magnetic structure, but is scattered over a large area. However, a more detailed analysis of the observations showed that the shape of the filament did not change during expansion. The apparent movement of plasma in different directions was due to its partial flowing back to the solar surface along the main axis of the filament simultaneously with the continuing upward expansion of the filament.

The use of model calculations for the second eruption (November 16, 2015) made it possible to estimate the area of the absorbing cloud in the microwave range, which amounted to $\approx 8 \%$ from the solar disk area. This value is close to the estimate of $10 \%$ obtained in [6], where an isolated negative burst was also observed as a result of absorption of the radiation of only the quiet-Sun regions by the matter of a large eruptive filament. In other studies [3, 5], where the cause for the depression of radio emission was the screening of both the quiet-Sun regions and the radio source, this area was $2-6 \%$ of the solar disk area. The estimated temperature of the eruptive filament matter $(9000 \mathrm{~K})$ and its mass $\left(3.3 \times 10^{15} \mathrm{~g}\right)$ agree with the estimates of temperatures and masses of large prominences, as well as with CME masses obtained in other 
Table 1. Information on the negative bursts identified with the events in the extreme ultraviolet

\begin{tabular}{|c|c|c|c|c|}
\hline No. & $\begin{array}{c}\text { Depression } \\
\text { observation } \\
\text { date and time }\end{array}$ & $\begin{array}{l}\text { Burst type, } \\
\text { observation frequencies, } \mathrm{GHz} \\
\text { (observatory) }\end{array}$ & $\begin{array}{l}\text { Events in EUV } \\
\text { and white light }\end{array}$ & $\begin{array}{l}\text { Flare class } \\
\text { (onset time) }\end{array}$ \\
\hline 1 & $\begin{array}{l}\text { Sept. 7, 2017, } \\
\text { 08:35-09:30 }\end{array}$ & $\begin{array}{l}\text { Isolated, with burst overlapping, } \\
2.7-8.8 \text { (San Vito) }\end{array}$ & $\begin{array}{l}\text { Two jets 08:37-09:00 } \\
\text { and 09:02-09:41 }\end{array}$ & C2.3 (09:16) \\
\hline 2 & $\begin{array}{l}\text { Sept. 7, 2017, } \\
\text { 10:22-11:20 }\end{array}$ & $\begin{array}{l}\text { Post-burst depression, } \\
2.7-8.8 \text { (San Vito) }\end{array}$ & $\begin{array}{l}\text { Filament eruption } \\
\text { (as a jet) and CME }\end{array}$ & M7.3 (10:11) \\
\hline 3 & $\begin{array}{l}\text { Sept. 8, 2017, } \\
\text { 01:00-02:00 }\end{array}$ & $\begin{array}{l}\text { Isolated, with burst overlapping, } \\
2-7.5 \text { (Nobeyama, UAPO, Lear- } \\
\text { month, SRG correlation curves) }\end{array}$ & $\begin{array}{l}\text { Filament activation } \\
\text { and partial eruption }\end{array}$ & C5.3 (01:36) \\
\hline 4 & $\begin{array}{l}\text { Sept. 8, 2017, } \\
03: 45-04: 30\end{array}$ & $\begin{array}{l}\text { Post-burst depression, } 2.7-8.8 \\
\text { (Nobeyama, Learmonth, SRG) }\end{array}$ & Jet $(03: 46-04: 30)$ & M1.2 (03:39) \\
\hline 5 & $\begin{array}{l}\text { Sept. 8, 2017, } \\
\text { 06:10-07:10 }\end{array}$ & $\begin{array}{l}\text { Post-burst depression, } 2.7-8.8 \\
\text { (Nobeyama, Learmonth, } \\
\text { San Vito, SRG) }\end{array}$ & Jet $(06: 10-07: 05)$ & C8.3 (05:31) \\
\hline 6 & $\begin{array}{l}\text { Sept. 8, 2017, } \\
\text { 10:50-11:20 }\end{array}$ & $\begin{array}{l}\text { Pre-burst depression, } 1.4158 .8 \\
\text { (San Vito) }\end{array}$ & $\begin{array}{l}\text { Filament activation (before 11:00) } \\
\text { and jet (11:00-11:40) }\end{array}$ & $\begin{array}{l}\text { C1.6 (10:45) } \\
\text { C1.7(11:21) }\end{array}$ \\
\hline 7 & $\begin{array}{l}\text { Sept. 8, 2017, } \\
\text { 12:10-12:50 }\end{array}$ & $\begin{array}{l}\text { Post-burst depression, } 2.7-8.8 \\
\text { (San Vito) }\end{array}$ & Jet $(12: 18-12: 55)$ & C5.9 (12:09) \\
\hline 8 & $\begin{array}{l}\text { Sept. 8, 2017, } \\
\text { 13:20-13:40 }\end{array}$ & Isolated, 2.7-15.4 (San Vito) & Filament activation & No flares \\
\hline 9 & $\begin{array}{l}\text { Sept. 8, 2017, } \\
\text { 12:10-12:50 }\end{array}$ & $\begin{array}{l}\text { Pre-burst depression, 5-15.4 } \\
\text { (San Vito) }\end{array}$ & Consecutive jets & M2.9 (15:09) \\
\hline
\end{tabular}

events [3]. In the SOHO LASCO CME Catalog, the mass of the CME was estimated at $1.7 \times 10^{16} \mathrm{~g}$, but an inaccuracy of this estimate was noted due to the fact that the ejection moved in a plane different from the SOHO sky plane.

Negative-burst events caused by the screening of near-limb radio sources by the jet matter were already reported in $[7,8]$. A similar picture was observed on September 7-9, 2017. It should be noted that all jets were in some way or another associated with flares. The type of negative burst depended on the class of the flare preceding the jet or following it. The depression of radio emission could even be an "isolated" negative burst if the flare class was low and the active region was close to the solar limb (such as, for example, the third negative burst in the event of September 8-9, 2017 or the radio emission depressions studied in [8]). Although, as a rule, such a burst is observed in the absence of any flares, as in the event of November 15$16,2015$.

It was previously noted that negative bursts in the microwave range are quite rare. The 12 depressions of radio emission observed during only three days in September 2017 show that they can occur quite often in the developing active region if it is located near the solar limb. In this case, the cause of depressions can be the activation of filaments or jets that are observed between the observer and the active region and partially obscure the radio sources located in it.

It was noted in [18] that when thermal radio radiation is absorbed by the low-temperature $\left(\sim 10^{4} \mathrm{~K}\right)$ material of the screen, the frequency range $<5 \mathrm{GHz}$ is the most preferable for observing negative bursts. At higher frequencies, the emission level usually decreased, but not below the pre-burst level.

An interesting case is when the depression of radio emission is present at high frequencies, but little manifested at low frequencies (for example, nos. 8 and 9 in Table 1). Figure 7 shows the records of the total radio flux on September 8, 2017 obtained at different frequencies in San Vito. Negative bursts at 11:00, 12:30, and 13:30 are visible at all frequencies, while the "preburst depression" after 14:00 is recorded only at frequencies $\geq 5 \mathrm{GHz}$. It should be noted that in this time interval, jets were observed one after another in the $304 \AA$ channel (Figs. 8c, 8d), although instability in the operation of the receiving equipment cannot be ruled out, which could manifest itself in a gradual decrease in the radio flux after 12:00 at frequencies $\geq 5 \mathrm{GHz}$.

What is the difference of the jets observed between 14:00 and 15:10 from others? It can be seen in Figs. $8 b-8 d$ that the direction of motion of the jets is 


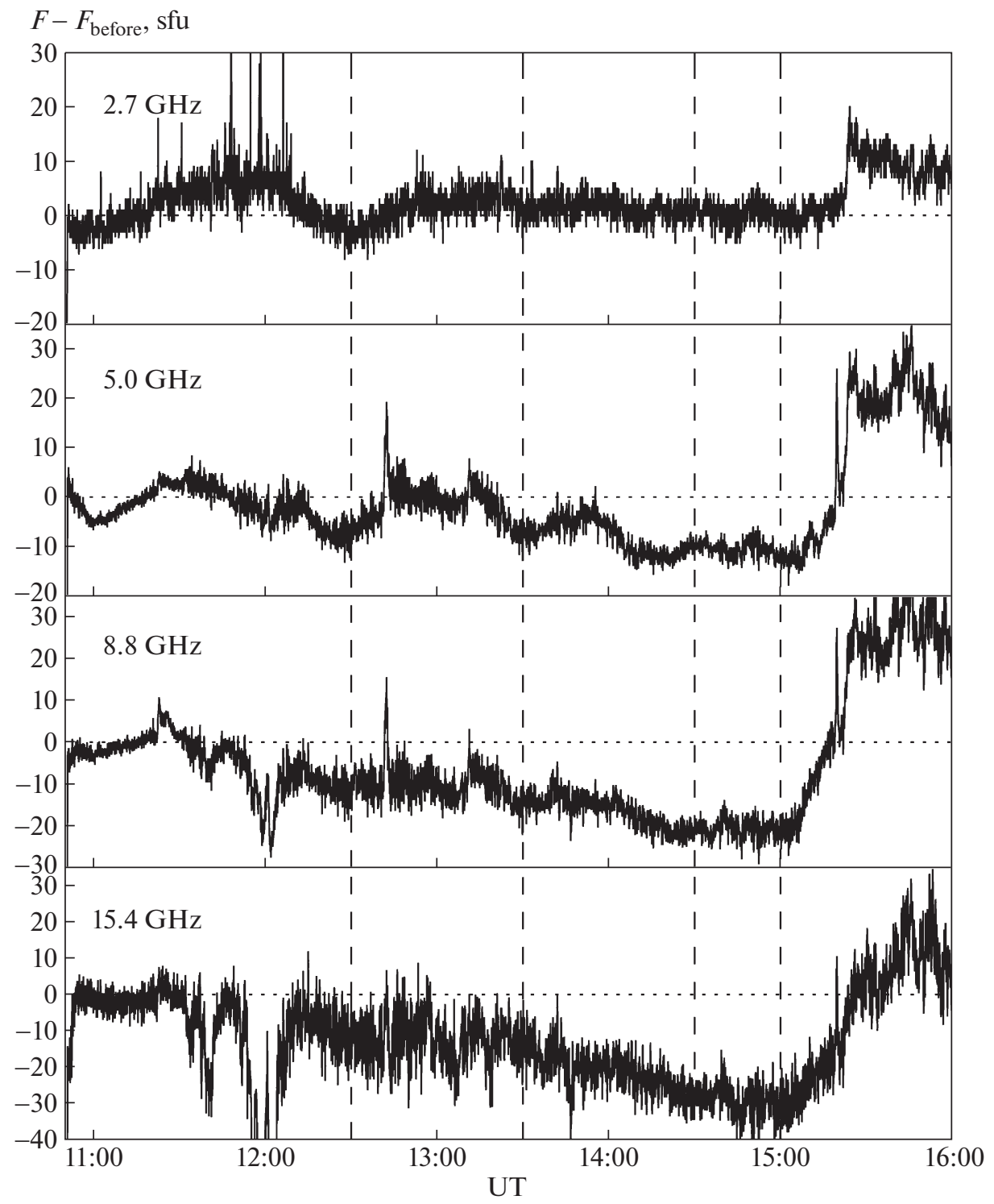

Fig. 7. Radio emission profiles on September 8, 2017 at different frequencies with the subtracted pre-burst level $F_{\text {before }}$ from the San Vito data. The vertical dashed lines mark the times corresponding to the images in Fig. 8.

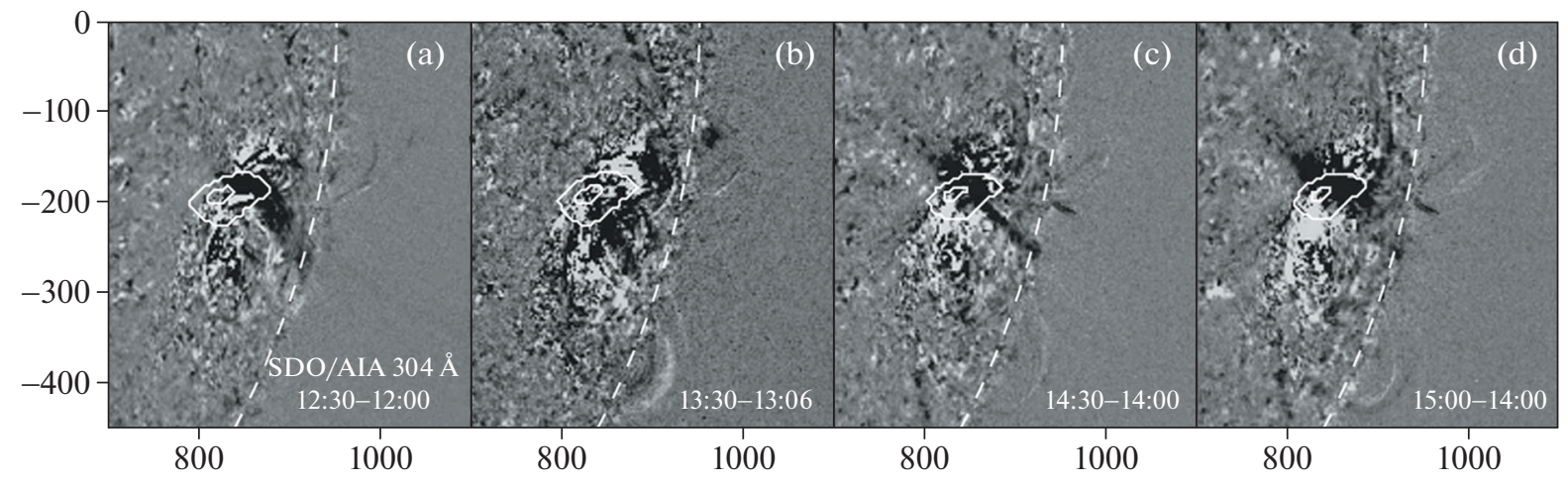

Fig. 8. Difference images of a part of the solar disk on September 8, 2017 according to the SDO/AIA data in the $304 \AA$ channel. The white outline shows the position of the radio source at the intensity level $[0.5 ; 0.9]$ from the maximum. The position determined from the images of the Nobeyama radioheliograph at a frequency of $17 \mathrm{GHz}$ on September 7, 2017 at 23:00 considering the Sun's rotation by the time indicated in the panels. The dashed arc marks the solar limb. 
such that their matter screened the brightest part of the radio source, in contrast to the jets in Figs. 8a, 6a, and 6b. Note that the images in Figs. $6 \mathrm{c}$ and $6 \mathrm{~d}$ also show the screening of the brightest part of the radio source, which corresponds to a decrease in the level of the radio flux at frequencies $>5 \mathrm{GHz}$ in Fig. 5 .

At high frequencies, radio sources are usually located above or between sunspots, while at low frequencies they are large (of the halo type). If the jet material screens a gyroresonance radio source (or a source above the neutral line) at high frequencies, the negative burst at these frequencies can be expected to be deeper. However, in this case, the area of the absorbing material may be insufficient for the appearance of a noticeable depression of radio emission at low frequencies, when the area of the absorber is small in comparison with the size of the radio source.

\section{CONCLUSIONS}

We studied the events in which negative bursts of different types were observed at different frequencies of the microwave range. In the event of November 1516,2015 , both radio bursts were of the rare "isolated" type and were associated with the eruptions of large quiet filaments between active regions in the southern hemisphere near the central meridian of the Sun. A detailed analysis of the observations in various spectral ranges showed that the cause for their occurrence was the matter of the eruptive filament absorbing the radiation from the regions of the quiet Sun and neighboring radio sources (November 15, 2015) and only the quiet-Sun regions (November 16, 2015). The estimates of the parameters of the absorbing matter on November 16, 2015 confirmed this conclusion.

For two negative bursts of the "pre-burst depression" type in the event of September 8-9, 2017, it was shown that they were associated with the appearance of jets in the active region or to the east of it. The active region had a complex magnetic configuration and was located near the western limb of the Sun. The material of the jets partially screened the radio sources (most likely gyroresonant) located in it, what caused negative bursts at a number of frequencies in the microwave range.

Comparison of the observations in the EUV and in the radio range on September 7-9, 2017 revealed that the appearance of jets in most cases corresponded to depressions in radio emission. At least 12 negative bursts were recorded at different observatories over the course of three days. This indicates that such events may not be as rare as previously thought. The burst type depended on the flare class and could even be "isolated" due to the position of the active region near the limb, when weak flares were present but had no response in the radio range. A previously unreported example of the occurrence of a deeper radio emission depression at high frequencies as compared to low frequencies was considered.

\section{ACKNOWLEDGMENTS}

The author is grateful to the colleagues from the Department of Radiophysics of ISTP SB RAS: Cand. Phys.Math. Sci. D.A. Zhdanov for kindly providing the spectropolarimeter data in the $4-8 \mathrm{GHz}$ frequency range, Dr. Phys.-Math. Sci. A.M. Uralov for valuable remarks and discussion of some points, and Dr. Phys.-Math. Sci. V.V. Grechnev for the possibility of using software from his IDL libraries. The author is grateful for free access to the NASA/SDO data and would like to thank the AIA scientific team, as well as the teams conducting observations at the US Air Force RSTN network and GOES satellites and the team that maintains the SOHO LASCO CME catalog at the NASA CDAW Data Center and the American Catholic University in cooperation with the US Naval Research Laboratory. We also thank the International Consortium for ensuring that observations with the Nobeyama Radioheliograph continued until the end of March 31, 2020. The Nobeyama Radio Polarimeters (NoRP) are operated by the Solar Science Observatory, a branch of the National Astronomical Observatory of Japan. The NoRP observational data are verified by the consortium that maintains NoRP for scientific purposes.

\section{OPEN ACCESS}

This article is licensed under a Creative Commons Attribution 4.0 International License, which permits use, sharing, adaptation, distribution and reproduction in any medium or format, as long as you give appropriate credit to the original author(s) and the source, provide a link to the Creative Commons license, and indicate if changes were made. The images or other third party material in this article are included in the article's Creative Commons license, unless indicated otherwise in a credit line to the material. If material is not included in the article's Creative Commons license and your intended use is not permitted by statutory regulation or exceeds the permitted use, you will need to obtain permission directly from the copyright holder. To view a copy of this license, visit http://creativecommons.org/licenses/by/4.0/.

\section{REFERENCES}

1. A. E. Covington, Solar-Geophys. Data 358, 20 (1974).

2. A. E. Covington, Solar Phys. 33, 439 (1973).

3. V. Grechnev, I. Kuzmenko, A. Uralov, I. Chertok, and A. Kochanov, Publ. Astron. Soc. Jpn. P65, SP10 (2013).

4. C. Sawyer, Solar Phys. 51, 203 (1977).

5. I. V. Kuzmenko, V. V. Grechnev, and A. M. Uralov, Astron. Rep. 53, 1039 (2009).

6. I. V. Kuzmenko and V. V. Grechnev, Solar Phys. 292, 143 (2017). 
7. V. V. Grechnev, S. V. Lesovoi, A. A. Kochanov, A. M. Uralov, et al., J. Atmos. Solar-Terr. Phys. 174, 46 (2018).

8. I. V. Kuzmenko, Solar-Terr. Phys. 6 (3), 23 (2020).

9. V. P. Maksimov and V. P. Nefedyev, Solar Phys. 136, 335 (1991).

10. A. Yu. Fedotova, A. T. Altyntsev, A. A. Kochanov, S. V. Lesovoi, and N. S. Meshalkina, Solar-Terr. Phys. 4 (3), 13 (2018).

11. V. V. Grechnev, A. M. Uralov, V. A. Slemzin, I. M. Chertok, I. V. Kuzmenko, and K. Shibasaki, Solar Phys. 253, 263 (2008)

12. I. V. Kuzmenko, F. A. Mikhalina, and B. A. Kapustin, Radiophys. Quantum Electron. 51, 905 (2008).
13. Y. Hou, T. Li, Z. Song, and J. Zhang, Astron. Astrophys. 640, A101 (2020).

14. Z. Song, Y. Hou, and J. Zhang, Astrophys. J. 892, 79 (2020).

15. D. A. Zhdanov and V. G. Zandanov, Centr. Eur. Astrophys. Bull. 35, 223 (2011).

16. V. N. Borovik, Lect. Notes Phys. 432, 185 (1994).

17. A. V. Kudryavtseva, I. I. Myshyakov, A. M. Uralov, and V. V. Grechnev, Solar-Terr. Phys. 7 (1), 3 (2021).

18. V. V. Grechnev, I. V. Kuzmenko, I. M. Chertok, and A. M. Uralov, Astron. Rep. 55, 637 (2011).

Translated by M. Chubarova 